\title{
Fiber's Impact on High-Sensitivity C-Reactive Protein Levels in Cardiovascular Disease
}

Renea L. Beckstrand

renea@byu.edu

Jennifer L. Butcher

Follow this and additional works at: https://scholarsarchive.byu.edu/facpub

Part of the Nursing Commons

\section{Original Publication Citation}

R. L. Beckstrand, J. L. Butcher, (29). Fiber's Impact on High-Sensitivity C-Reactive Protein Levels in Cardiovascular Disease.

\section{BYU ScholarsArchive Citation}

Beckstrand, Renea L. and Butcher, Jennifer L., "Fiber's Impact on High-Sensitivity C-Reactive Protein Levels in Cardiovascular Disease" (2009). Faculty Publications. 55.

https://scholarsarchive.byu.edu/facpub/55

This Poster is brought to you for free and open access by BYU ScholarsArchive. It has been accepted for inclusion in Faculty Publications by an authorized administrator of BYU ScholarsArchive. For more information, please contact ellen_amatangelo@byu.edu. 


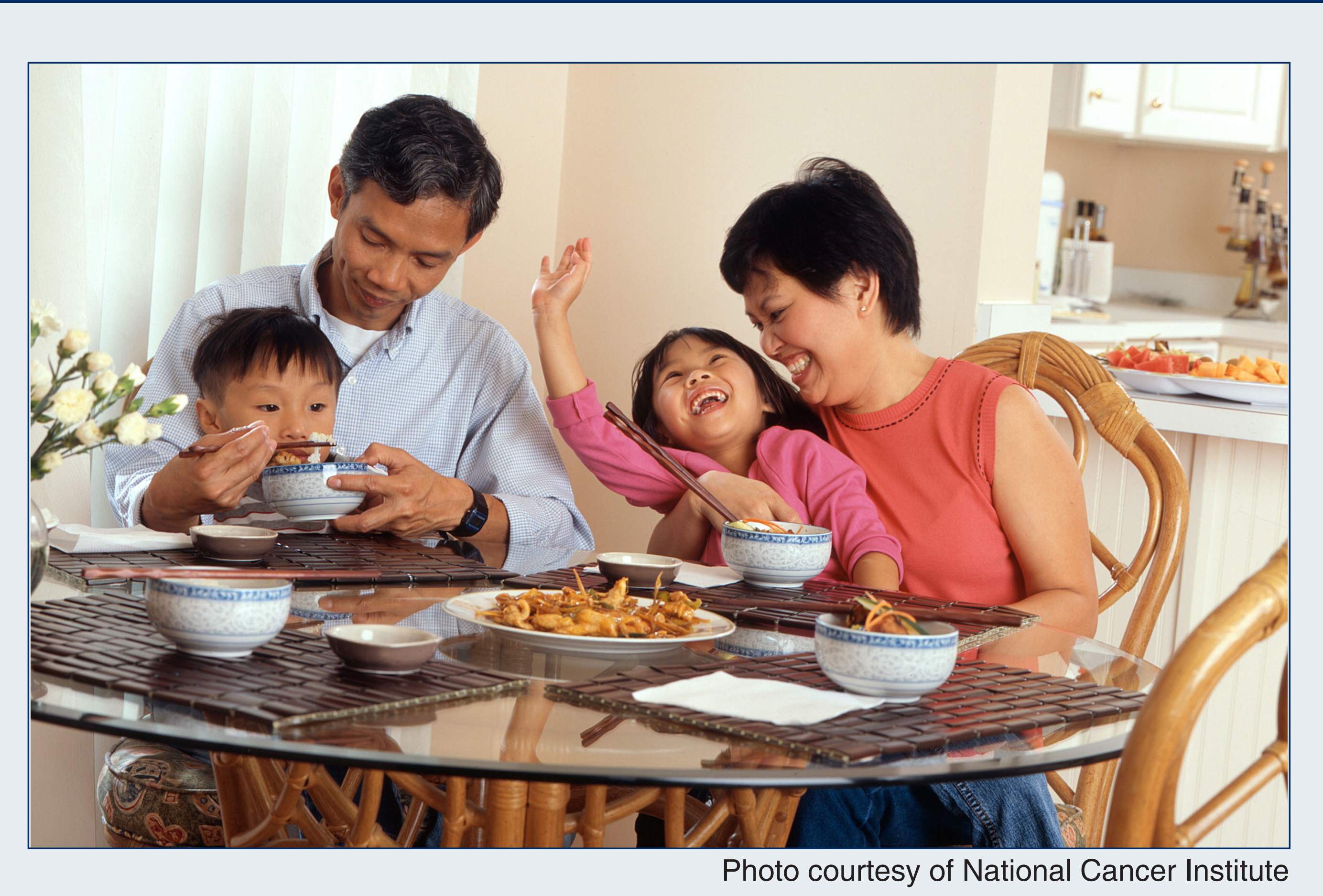

Purpose

To determine if increased dietary or supplemental intake of fiber slows or prevents inflammation as evidenced by hs-CRP values.

\section{Background}

High sensitivity-CRP is the most sensitive lab test to detect inflammation in the body and helps predict cardiovascular risk.

- $<1 \mathrm{mg} / \mathrm{L}=$ low level prediction of CV events

- 1-3 mg/L = moderate level prediction of CV events

- $>3 \mathrm{mg} / \mathrm{L}=$ high risk for future $\mathrm{CV}$ events

\section{Data Sources}

CINAHL, Medline, Health Source: Nursing/Academic Edition, and the Cochrane Library.

\begin{tabular}{|l|l|}
\hline Body Mass Index \\
\hline Normal Weight & $18.5-24.9 \mathrm{~kg} / \mathrm{m} 2$ \\
\hline Overweight & $25.0-29.9 \mathrm{~kg} / \mathrm{m} 2$ \\
\hline Obese & $30 \mathrm{~kg} / \mathrm{m} 2$ and above \\
\hline & $\begin{array}{l}\text { National Heart Lung and Blood } \\
\text { lnstitute (2008) }\end{array}$ \\
\hline
\end{tabular}

\section{Evidence}

-Elevated hs-CRP levels have also been related to insulin resistance and metabolic syndrome

- Pharmacological agents, such as statin drugs, have been shown to decrease hs-CRP levels

- Dietary fiber, a non-pharmacological remedy, has also been shown to decrease hs-CRP levels

\begin{tabular}{|c|c|c|c|}
\hline & $\begin{array}{l}\text { Food } \\
\text { Sources }\end{array}$ & Serving Size & $\begin{array}{l}\text { Amount of } \\
\text { fiber grams } \\
\text { (g) per } \\
\text { serving }\end{array}$ \\
\hline \multirow{10}{*}{$\begin{array}{l}\text { Soluble } \\
\text { Sources }\end{array}$} & oatmeal & $1 / 2$ cup, dry & $4 \mathrm{~g}$ \\
\hline & oatbran & 1 cup, cooked & $5.7 \mathrm{~g}$ \\
\hline & lentils & 1 cup, cooked & $15.6 \mathrm{~g}$ \\
\hline & nuts (almonds) & 1 ounce & $3.5 \mathrm{~g}$ \\
\hline & beans (black) & 1 cup, cooked & $15.0 \mathrm{~g}$ \\
\hline & peas (frozen) & 1 cup, cooked & $8.8 \mathrm{~g}$ \\
\hline & apples & 1 apple & $3.3 \mathrm{~g}$ \\
\hline & pears & 1 pear & $5.1 \mathrm{~g}$ \\
\hline & strawberries & 1 cup & $3.3 \mathrm{~g}$ \\
\hline & blueberries & 1 cup, raw & $3.5 \mathrm{~g}$ \\
\hline \multirow[t]{13}{*}{$\begin{array}{l}\text { Non-soluble } \\
\text { Sources }\end{array}$} & $\begin{array}{l}\text { whole wheat } \\
\text { bread }\end{array}$ & 1 slice & $1.9 \mathrm{~g}$ \\
\hline & barley & 1 cup, raw & $31.2 \mathrm{~g}$ \\
\hline & couscous & 1 cup, cooked & $2.2 \mathrm{~g}$ \\
\hline & brown rice & 1 cup, cooked & $3.5 \mathrm{~g}$ \\
\hline & bulgur & 1 cup, dry & $25.6 \mathrm{~g}$ \\
\hline & $\begin{array}{l}\text { whole grain } \\
\text { breakfast cereals } \\
\text { (Kellogg's All- } \\
\text { Bran) }\end{array}$ & $1 / 2$ cup & $8.8 \mathrm{~g}$ \\
\hline & wheat bran & 1 cup, crude & $25 \mathrm{~g}$ \\
\hline & $\begin{array}{l}\text { seeds } \\
\text { (sunflower, dry } \\
\text { roasted, salted) }\end{array}$ & $1 / 4$ cup & $2.9 \mathrm{~g}$ \\
\hline & carrots & 1 cup, raw & $3.1 \mathrm{~g}$ \\
\hline & cucumbers & $\begin{array}{l}1 \text { cup, peeled, } \\
\text { raw }\end{array}$ & $0.8 \mathrm{~g}$ \\
\hline & zucchini & 1 cup, cooked & $3 \mathrm{~g}$ \\
\hline & celery & 1 cup, raw & $1.9 \mathrm{~g}$ \\
\hline & tomatoes & 1 tomato, raw & $1.5 \mathrm{~g}$ \\
\hline
\end{tabular}

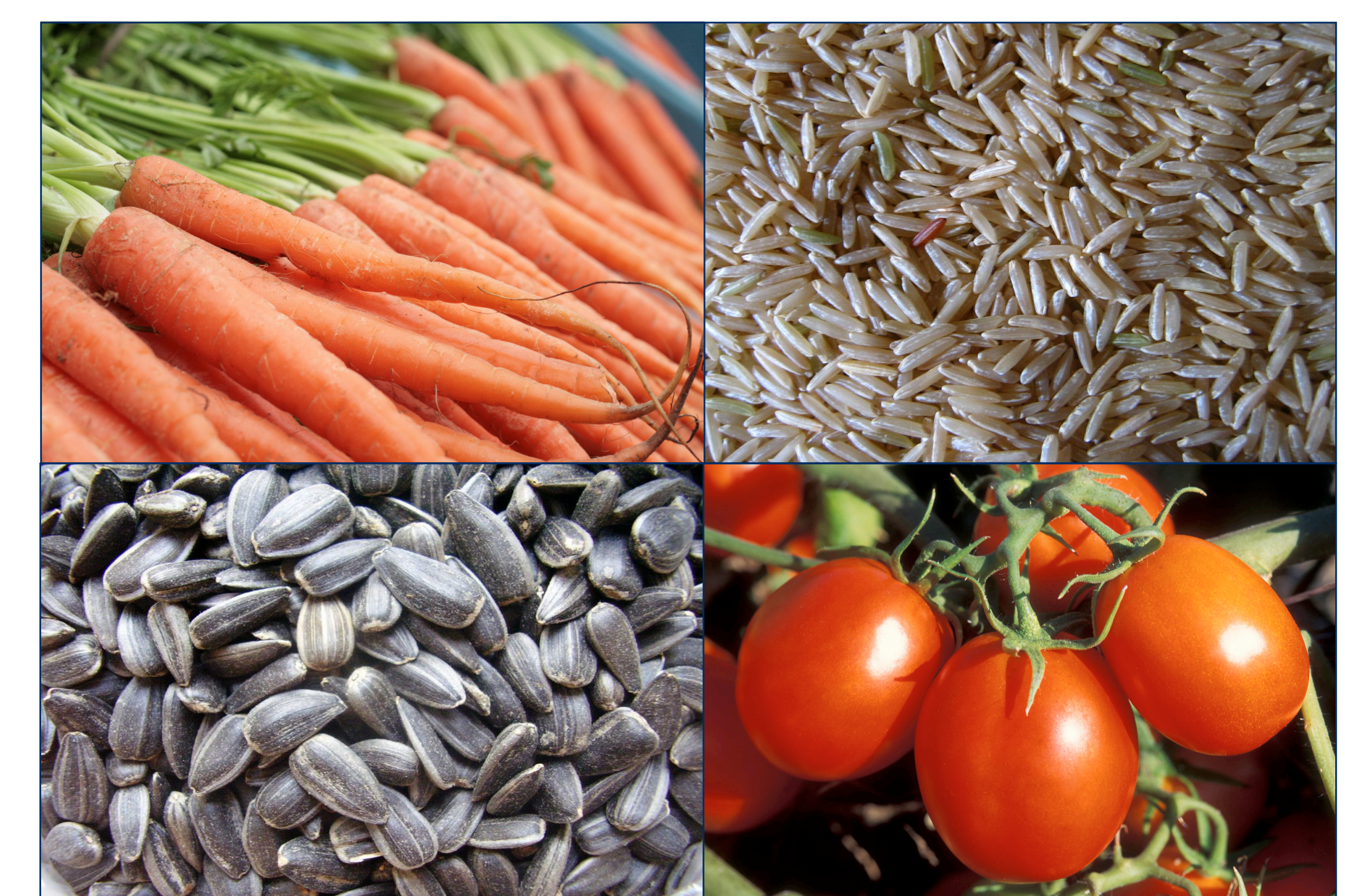

\section{Findings}

Evidence from this review suggested significant associations between fiber consumption and decreased risk for cardiovascular disease as evidenced by a decrease in hs-CRP levels. Six of the 7 articles reviewed showed statistically significant decreases in hs-CRP levels as dietary or supplemental intake of fiber was increased.

\section{Implications}

Fiber in the diet may play a strong role in cardiovascular health as evidenced by 6 clinical trials completed using amount of fiber intake in relation to inflammation, particularly hs-CRP levels. Patients need to be educated regarding a high fiber diet, either by dietary or supplemental means, using the recommended 25 - 30 grams of fiber per day.

\section{Key Words}

Fiber, Inflammation, Cardiovascular disease, Literature Review. 